\title{
Analysing, Interpreting, and Testing the Invariance of the Actor-Partner Interdependence Model iㅣ 웅
}

\author{
Alexandre Gareau $^{\mathrm{a}, \bigotimes}$, Josee Fitzpatrick ${ }^{\mathrm{a}}$, Patrick Gaudreau ${ }^{\mathrm{a}} \&$ Marie-France Lafontaine $^{\mathrm{a}}$ \\ ${ }^{\mathrm{a}}$ School of psychology; University of Ottawa
}

\begin{abstract}
Although in recent years researchers have begun to utilize dyadic data analyses such as the actor-partner interdependence model (APIM), certain limitations to the applicability of these models still exist. Given the complexity of APIMs, most researchers will often use observed scores to estimate the model's parameters, which can significantly limit and underestimate statistical results. The aim of this article is to highlight the importance of conducting a confirmatory factor analysis (CFA) of equivalent constructs between dyad members (i.e. measurement equivalence/invariance; $\mathrm{ME} / \mathrm{I})$. Different steps for merging CFA and APIM procedures will be detailed in order to shed light on new and integrative methods.
\end{abstract}

Keywords $\square$ Actor-partner interdependence model, dyadic data analysis, invariance testing, CFA, methodology. Tools $\backsim$ MPLUS.

agare104@uottawa.ca

AG: 0000-0003-2517-5109; JF: 0000-0002-6450-1408; PG: 0000-0003-4094-5423; MFL: 0000-00034185-6326

10.20982/tqmp.12.2.p101

Acting Editor $\square$ Denis Cousineau (Université d'Ottawa)

Reviewers

- One anonymous reviewer

\section{Introduction}

In a previous article (Fitzpatrick, Gareau, Gaudreau, \& Lafontaine, 2015), we explained how to test the actor-partner interdependence model (APIM) with structural equation modeling (SEM), with all variables treated as continuous manifest/observed variables. Following this strategy has certain benefits, such as reducing the complexity of a model, but also has certain limitations such as losing specific information from each item in the computation of the mean score (i.e., $X 1, X 2, Y 1$, and $Y 2$ ). One way to take full advantage of the available data and address this limitation is to create latent variables by incorporating the features of a factor analytical model within the APIM (Bolger \& Shrout, 2007; Cook, 1994; Orth, 2013). This brings to light one important question that has yet to be fully addressed and incorporated in the APIM literature: Are the equivalent factors across the two partners really measuring the same thing? Given that in the APIM analysis, the data has a dyad-level structure instead of an individuallevel structure, the factorial constructs are exposed to legitimate query on their actual comparability. The question is thus simple: Is the factorial structure of concept $\mathrm{X}$ the same across the two members of the dyad? If this is not the case, the direct interpretability of the results of the APIM is minimized. The solution for such an important issue is known as the measurement of equivalence/invariance (ME/I). As such, the purpose of this article is to presents how to incorporate latent variables and to estimate their dyadic invariance in an APIM. We propose an extension of the APIM in order to fully incorporate the many advantages offered by SEM by modeling latent variables rather than manifest variables in order to take into account measurement error.

\section{Factor analysis general assumptions}

Many textbook or articles already described the basics of factor analysis (e. g., Brown, 2006; Yong \& Pearce, 2013). Generally, factor analytical strategy is rooted in classical test theory and is guided by the overarching principle that:

$$
\text { Observed score }=\text { True score }+ \text { error }
$$

Accordingly, in a factor analysis model, measurement error is accounted for, which allows for a more precise estimation of the effects. There are currently two main methods that use factor analytical strategies, namely the exploratory factor analysis (EFA) and the confirmatory fac- 
tor analysis (CFA). EFA is mostly used in the early development stage of an instrument for investigating the number of possible factors existing within a set of items whereas CFA is driven with a priori knowledge about the factor structure and the number of factors. In the CFA framework, different hypotheses can be tested with regards to the factorial structure as well as the factorial equality of two constructs, which may be of greater interest (Vandenberg \& Lance, 2000). For the present text, we recommend that readers already have a general comprehension of factor analysis and SEM procedures (see Gunnell, Gareau, \& Gaudreau, 2016; Weston \& Gore, 2006).

\section{Measurement Equivalence/Invariance with dyadic data}

The measurement of equivalence and invariance has been employed in a variety of disciplines and is seen by some as a necessary step prior to the specification of any statistical model that aims at comparing groups and/or longitudinal data (Brown, 2006). Variance between members (i.e., within-dyad) in dyadic data analysis needs to be tested for its equality. Researchers can use two different approaches to test the invariance of a latent variable across two groups: a multi-group approach or a single sample approach, in which all the observed variables of the two partners are dealt with simultaneously (Brown, 2006). In the case of the APIM, it would be preferable to use the single sample approach because the members of the distinguishable groups (e.g., male and female) are not independent. This approach, which is comparable to invariance testing of longitudinal data, is advantageous because it can estimate the correlation between errors of the items used respectively to measure the first and second partner's (i.e., in this case a male and female) assessed variables. Thus, it can ensure that non-independence of the data is taken into consideration in the model. In this approach, the parameter estimates of the latent variable of the male $(X 1)$ and the latent variable of the female $(X 2)$ can be constrained to equality to examine the extent to which the variable can be considered invariant across the two members of the dyad. However, an important disadvantage of the single sample approach is the large size of the covariance matrix, which can lead to poor model fit and model convergence problems (Brown, 2006). Larger samples are therefore required with a single sample approach.

\section{Current approach for testing $M E / I$}

A stepwise procedure is traditionally implemented to test measurement invariance, with steps going from a least restricted model to increasingly restrictive constraints. Different researchers have prescribed a series of different steps in order to successfully estimate the measurement invariance of a construct. In their comprehensive and authoritative review of the measurement invariance literature, Vandenberg and Lance (2000) presented an elaborate series of eight steps that have been found to be consistent throughout studies; the first five steps pertain to aspects of measurement invariance and the following three pertain to the structure. Our approach is largely inspired by the recommendations of Vandenberg and Lance (2000) but the first step (i.e., omnibus test of the equality of covariance matrices across groups; $\Sigma^{g}=\Sigma^{g^{\prime}}$ ) is omitted in our article because it can only be implemented in a multi-group approach. The following four steps pertaining to aspects of measurement invariance will be used within the APIM framework. Vandenberg and Lance's (2000) steps pertaining to structure (i.e., steps 6-8) have also been omitted since they cannot be performed in our case, again because only one covariance matrix is used. For the practical purposes of the present article, we decided to add one last step (i.e., step 5) for decision-making purposes (the statistical symbols used throughout this article are from the LISREL matrix notation).

1. Identification of configural invariance; verification of an identical pattern of fixed and free factor loadings across groups.

2. Identification of metric invariance; verification of the equality on the loadings of items across genders (i.e., $\Lambda^{g}=\Lambda^{g^{\prime}}$ ).

3. Identification of scalar invariance; verification of the equality on the intercept of items across genders (i.e., $\tau^{g}=\tau^{g^{\prime}}$ ).

4. Identification of uniqueness invariance; verification of the equality on the residual items across genders (i.e., $\left.\Theta^{g}=\Theta^{g^{\prime}}\right)$.

5. Making a decision from the ME/I sequence.

Most research using the APIM model focuses on the actor and partner effects. Extending the APIM to latent variables offers the opportunity to examine whether the latent variables of the two partners (e.g., male and female) are equivalent. Such a demonstration would be important before determining whether the effects (e.g., actor and partner effects) of one partner are significantly stronger than the effects of the other partner. Failure to demonstrate sufficient evidence of measurement invariance across the two partners would compromise the comparison of the effects, which is at the heart of the APIM analyses.

Comparisons of the nested models. The hierarchical approach described above implies that new equality constraints are added at each step of the invariance analysis. Therefore, the models tested at each step are nested in a way to proceed from a less constrained model (i.e., with less degrees of freedom) to a more constrained model (i.e., with more degrees of freedom). When comparing nested 
models in a sequence, only using the $\chi^{2}$ difference test can lead to important bias in accordance with its known sensitivity to sample size. In order to add credence in the comparison of models, assessing the differences/changes in CFI has been recommended as a relevant addition to the $\chi^{2}$ difference test (Cheung \& Rensvold, 1999). On this topic, Meade, Johnson, and Braddy (2008) have determined that changes greater than .002 in CFI could indicate notable differences between nested models. In addition, we propose to use Satorra and Bentler (1994) scaled $\chi^{2}$ that corrects for non-normality. However, this statistic cannot be used in a normal $\chi^{2}$ difference test because the distribution of the actual difference does not follow the $\chi^{2}$ distribution. To circumvent this issue, Satorra and Bentler (2001) created formulas that have been integrated into an online calculator by Colwell (2013). Maximum likelihood robust estimation is needed when achieving this test and will be requested in MPLUS using the estimator command. The calculated $\Delta$ Satorra-Bentler $\chi^{2} \mathrm{p}$-value can then be obtained in a $\chi^{2}$ table or with Soper (2013) calculator if you wish to obtain the exact $\mathrm{p}$-value.

\section{Participants Used for the Example}

To clearly illustrate our recommended approach, we used a sample of 246 individuals from 123 adult heterosexual couples aged between 21 and 80 years old for men (M $=33.44, \mathrm{SD}=11.30$ ) and between 20 and 79 years old for women $(\mathrm{M}=31.25, \mathrm{SD}=9.94)$. Participants were community-based couples who had been involved in their romantic relationship with their partner for at least one year. We used data collected through questionnaires answered by these couples to examine whether one's own and partner's sensitivity could predict relationship satisfaction. Sensitivity was measured using the sensitivity scale of the Caregiving Questionnaire on a 6-point Likert scale (CGQ; Kunce \& Shaver, 1994). An example of an item would be "I am very attentive to my partner's nonverbal signals for help and support" (Male $\alpha=.86$; Female $\alpha=.83)$. Relationship satisfaction was measured using the brief validated version of the Dyadic Adjustment Scale on a 6-point Likert scale (DAS-4; Sabourin, Valois, \& Lussier, 2005). The DAS-4 is widely used to assess the degree of relationship satisfaction for individuals in marital relationships. An example of an item would be "Do you confide in your mate?" (Male $\alpha=.86$; Female $\alpha=.76$ ). The corresponding data file is available on the journal's website. In the following article, we used MPLUS 6.12 (Muthén \& Muthén, 2012) to conduct all analyses; all analyses could also be implemented in other SEM modeling programs.

\section{Measurement invariance steps with MPLUS}

In this article, we propose and illustrate how a measurement model can be initially tested with a confirmatory factor analysis (CFA) before estimating the APIM within the confines of a full SEM model with latent variables. We also highlight and illustrate how equality constraints can be implemented to estimate whether the latent variables are equivalent across both members of a distinguishable dyad (e.g., gender invariance). Note that the following steps are only valid for continuous data analysis. Ordinal data invariance testing requires a different set of steps that will not be explained in the current article (see Millsap \& YunTein, 2004).

Step 1: Configural model. The configural invariance model goal is to test the equivalence of the factor structure. Thus, the hypothesis put to the test is that the congeneric model holds between the two constructs. The configural model specifications are the following: (a) Loadings are all freely estimated, except that the first item of each construct needs to have its loading fixed to 1.0 and its (b) intercept to 0.0 in order to define the latent variable's scale and mean, respectively; (c) the mean, variance, and covariance of each created factor needs to be freely estimated; (d) correlations between equivalent items residuals also need to be estimated (e.g., $\delta_{11} \leftrightarrow \delta_{21}$ ) to control for nonindependence. Configural invariance must be established to proceed to the subsequent step, which ultimately means that a model imposing the same number of factors (with the exact fixed and freed parameters) is equally acceptable for both members of the dyad. Listing 1 indicates how this model is fitted to data using MPLUS. The results of the configural model showed good fit indices as seen in Table 1.

Step 2: Metric model $\left(\Lambda^{g}=\Lambda^{g^{\prime}}\right)$. The hypothesis for the metric model is that the strength of the factor loadings for equivalent items are invariant across members (e.g., $\lambda_{12}=\lambda_{22}$ ). Partial invariance of the loadings is a minimal requirement to proceed to the next step. Failure to demonstrate partial metric invariance could indicate that several items are better indicators of the latent variable for one partner compared to the other. Listing 2 shows how the model is fitted to the data.

In our example, the Satorra-Bentler scaled $\chi^{2}$ difference tests did not indicate a significant change but the $\triangle C F I$ was above .002, as seen in line Step 2 of Table 1 . We further investigated the possible misspecifications of the model with modification indices $\left(\Delta d f=1, \chi^{2}=3.84, p=\right.$ .05 ) and by examining meaningful loading differences in the configural model. This is achieved by adding the following OUTPUT statement as given in Listing 3.

As seen in Table 1, line Step 2, the modification index for the loading of the Y construct item 3 ( $\chi^{2}=4.42, p<$ 
Listing 1 - Analysis and Model command for the configural model of Step 1.

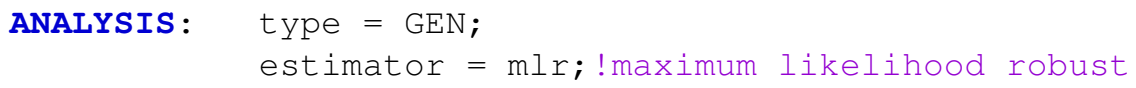


Table 1 a Test of measurement invariance/equivalence and the APIM structural models

\begin{tabular}{|c|c|c|c|c|c|c|c|c|c|c|}
\hline Models & $\mathrm{df}$ & $\mathrm{SB}_{\chi^{2}}$ & SRMSR & RMSEA & TLI & CFI & $\Delta$ CFI & $\Delta \mathrm{df}$ & $\Delta \mathrm{SB}_{\chi^{2}}$ & $\mathrm{CD}$ \\
\hline \multicolumn{11}{|l|}{ Measurement Invariance } \\
\hline Step 1: Configural model & 90 & 98.889 & .050 & .028 & .983 & .987 & & & & \\
\hline Step 2: Metric model $\left(\Lambda^{g}=\Lambda^{g^{\prime}}\right)$ & 96 & 108.223 & .071 & .032 & .978 & .982 & .005 & 6 & 8.894 & 1.307 \\
\hline $\begin{array}{l}\text { Step 2a: loading constraint re- } \\
\text { lease Y item3 }\end{array}$ & 95 & 104.542 & .061 & .029 & .982 & .986 & .001 & 5 & 5.641 & \\
\hline Step 3: Scalar model $\left(\tau^{g}=\tau^{g^{\prime}}\right)$ & 101 & 116.129 & .077 & .035 & .974 & .978 & .008 & 6 & $11.366 \dagger$ & 1.141 \\
\hline $\begin{array}{l}\text { Step3a: Intercept constraint re- } \\
\text { lease Y item3 }\end{array}$ & 100 & 111.470 & .065 & .031 & .980 & .983 & .003 & 5 & 7.1314 & 0.946 \\
\hline $\begin{array}{l}\text { Step 4: Invariant uniqueness } \\
\operatorname{model}\left(\Theta_{\delta}^{g}=\Theta_{\delta}^{g^{\prime}}\right)\end{array}$ & 108 & 142.638 & .107 & .051 & .944 & .949 & .034 & 8 & $24.033^{*}$ & 1.653 \\
\hline $\begin{array}{l}\text { Step4a: Uniqueness constraint } \\
\text { release Y item4 }\end{array}$ & 107 & 124.566 & .109 & .037 & .971 & .974 & .009 & 7 & 11.030 & 1.855 \\
\hline $\begin{array}{l}\text { Step4b: Uniqueness constraint } \\
\text { release Y item1 }\end{array}$ & 106 & 117.501 & .079 & .030 & .981 & .983 & .000 & 6 & 6.209 & 1.515 \\
\hline \multicolumn{11}{|l|}{ Structural model (APIM) } \\
\hline Step 1: Basic APIM (model 1) & 106 & 117.501 & .079 & .030 & .981 & .983 & & & & \\
\hline $\begin{array}{l}\text { Step 2: With the } \mathrm{k} \text { parameters } \\
\text { (model 2) }\end{array}$ & 106 & 117.501 & .079 & .030 & .981 & .983 & & & & \\
\hline Step2a: With k1@1.5 and k2@0.5 & 108 & 116.167 & .080 & .025 & .987 & .988 & .005 & 2 & 0.0263 & 1.812 \\
\hline
\end{tabular}

Note. Note. Significance of the $\Delta S B_{\chi^{2}}$ is indicated with $*: p<.05 ; \dagger: p<.08 . \mathrm{SB}_{\chi^{2}}=$ Satorra-Bentler scaled $\chi^{2}$ difference test; SRMSR = standardized root mean squared residual; RMSEA = root mean squared error of approximation; $\mathrm{TLI}=$ Tuker-Lewis index; CFI = comparative fit index; $\mathrm{CD}=$ Scaling correction .

.05) was significant and the loadings from the configural model suggested a meaningful difference across the two partners $\left(\lambda_{13}(\right.$ male $)=.49$ vs. $\lambda_{23}($ female $\left.)=.75\right)$. We thus proceeded to release this equality constraint. Partial invariance should be tested at the metric level only for a minority of indicators and with strong theoretical guidance (Vandenberg \& Lance, 2000). This new model was not significantly different based on the $\Delta \mathrm{SB}_{\chi^{2}}$ and $\Delta C F I$. Full invariance can be assumed for sensitivity (X construct) whereas partial invariance can be assumed for the relationship satisfaction (Y construct).

Step 3: Scalar model $\left(\tau^{g}=\tau^{g^{\prime}}\right)$. In this step, the intercepts of each item are constrained across members (e.g., $\tau_{12}=\tau_{22}$ ). In line with item-response theory, intercepts can be interpreted as the value of an item when the latent factor is 0 . Therefore, the intercepts offer information about occurrence of a systematic bias (e.g., response style or difficulty) in the manner the question was answered. Because the intercept of the first item is already fixed to 0.0 (step 1), only the other items need to be forced to equality across members. This is done using Listing 4 .

As seen in Table 1, Step 3a, in our example, the scalar model showed a marginally significant difference in the $\Delta \mathrm{SB}_{\chi^{2}}$ and a $\Delta \mathrm{CFI}$ higher than .002. The modification index for the intercept of item 3 from the $Y$ latent variable reached statistical significance $\left(\chi^{2}=5.31, p<.05\right)$. After releasing this equality constraint, the model was not significantly different from the metric invariance model. Overall, these results provide evidence for the full scalar invariance of the sensitivity latent variable and the partial scalar invariance of the relationship satisfaction latent variable.

Step 4: Invariant uniqueness model $\left(\Theta^{g}=\Theta^{g^{\prime}}\right)$. For this model, the unique/residual /error variance of each item is set to equality across members (e.g., $\delta_{11}=\delta_{21}$ ). In other words, this step controls for the specific effect occurring in each item across dyads. The residual represents the part of the item that is not explained by the latent variable. Putting equality constraints on the residual thus ensures that the variance of each item explained by the latent variables is the same for both partners. Listing 5 incorporate these constraints.

In our example, this model showed a significant difference in the $\Delta \mathrm{SB} \chi^{2}$ and a $\Delta \mathrm{CFI}$ over .002, as seen in Table 1, line Step 4 . The modification index for the uniqueness of item 4 (Y latent variable) was significant ( $\chi^{2}=$ $15.31, p<.05)$. After dropping this equality constraint, the model significantly improved but $\Delta$ CFI was still above .002 , as seen in Table 2, Step 4a. The modification index for the residuals of item 3 (Y latent variable) was also significant $\left(\chi^{2}=9.14, p<.05\right)$. We released this last equal- 
Listing 2 a Model command for the metric model of Step 2. The loading parameters in Listing 1 must be replaced by these commands.

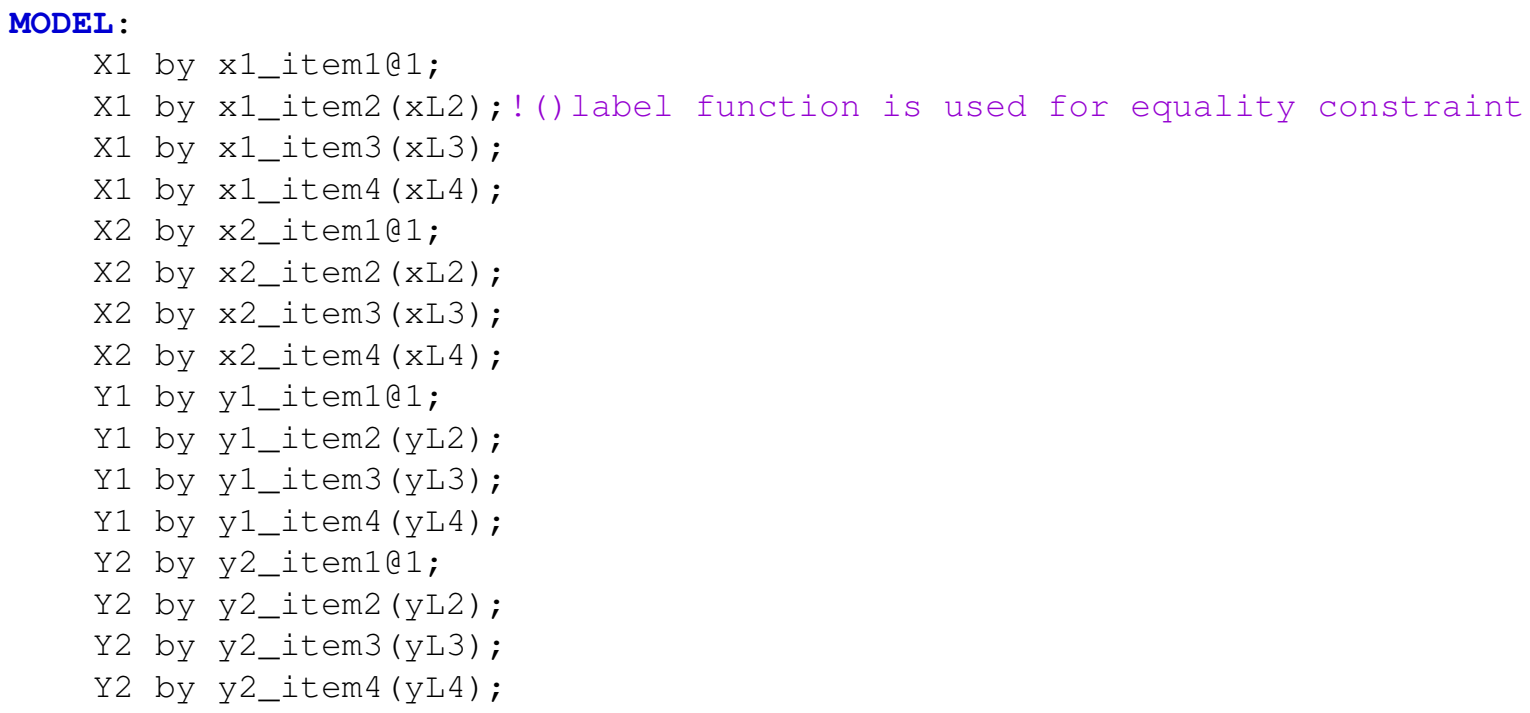

Listing 3 OUTPUT command of Step 2

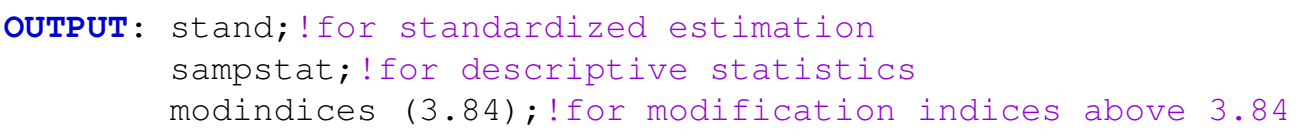

ity constraint and retained this last model, as indicated in Table 1, Step 4b. Overall, full residual invariance can be assumed for sensitivity (X construct) whereas partial residual invariance can be assumed for relationship satisfaction (Y construct).

Step 5: Making a decision from the ME/I sequence. The question that was driving this sequence of testing was one of major importance: to what extent is the factor structure of the psychological constructs (i.e., sensitivity and relationship satisfaction) similar across partners? If the factor structure was not similar, the results from the subsequent APIM would need to be interpreted with extreme caution as key parameters of the measurement model are not directly comparable and equivalent across the two partners. As a result, observed differences in the actor and/or partner effects of the male and the female could be attributable to either true difference or measurement non-invariance. Therefore, we could not attest to the patterns or any cross-over prediction; as aptly said by Vandenberg and Lance (2000), "Comparisons of apples to apples are meaningful. Comparisons of apples to sandwiches to sand wedges are not.” (p. 40). Following this line of reasoning, the ME/I sequence is an essential prerequisite to any trustworthy APIM estimation. SEM with latent variables becomes an indispensable tool to evaluate the extent to which estimates from the APIM can generate trustworthy conclusions. Evaluating the equivalence of measures across partners will rarely be a dichotomous black or white judgement. However, reporting - rather than not testing - the area of non-invariance is likely to help readers to better evaluate the measurement context under which the actor and partner effects were produced. It will also inform researchers about problematic items that might need to be reformulated to avoid gender-specific biases in future studies. In our example, only few parameters of the $\mathrm{Y}$ construct (i.e., relationship satisfaction) were significantly different (i.e., non-invariant) across partners at each step (one third of the loadings; one third of the intercepts; two out of four uniqueness parameters). The relationship satisfaction latent variable can therefore be considered sufficiently invariant across dyads to enable a decent estimation of the APIM. In contrast, none of the parameters significantly differed across the partners for the $\mathrm{X}$ construct (i.e., sensitivity). The final invariance measurement model can then be used as a foundation for testing the APIM with latent variables, as presented in our past article (Fitzpatrick 
Listing 4 ॥ Model command for the scalar model of Step 3. The intercept parameters in Listing 1 should be replaced by these commands.

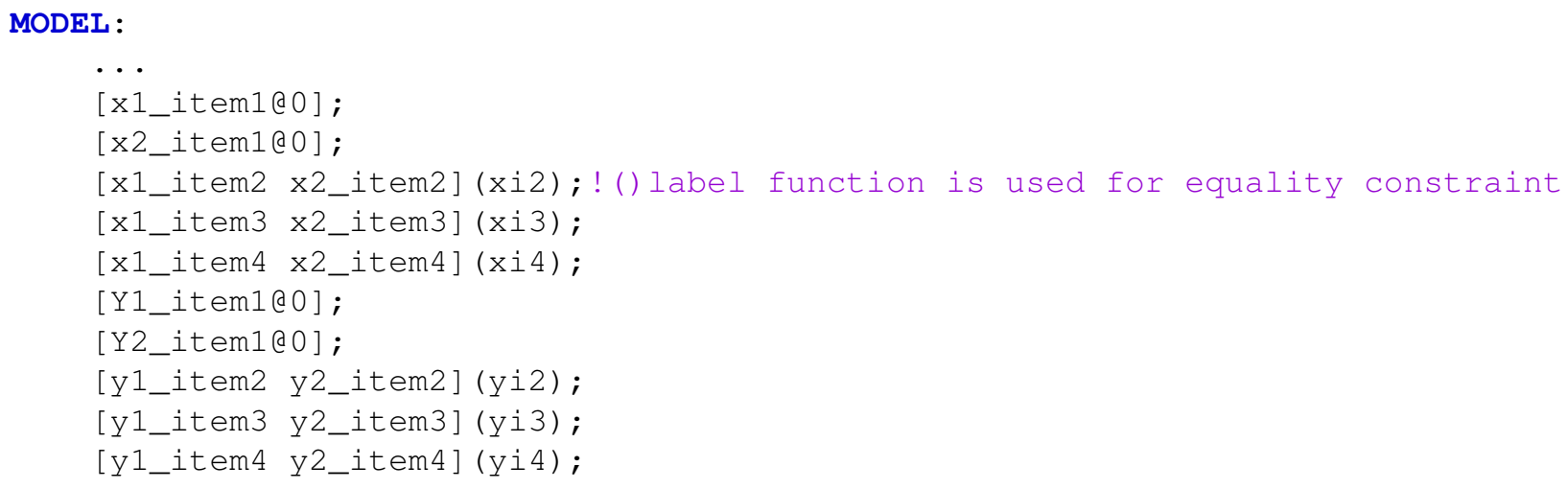

Listing 5 - Model command for the uniqueness model of Step 4. These commands are added to the Model command of Listings 2 and 4.

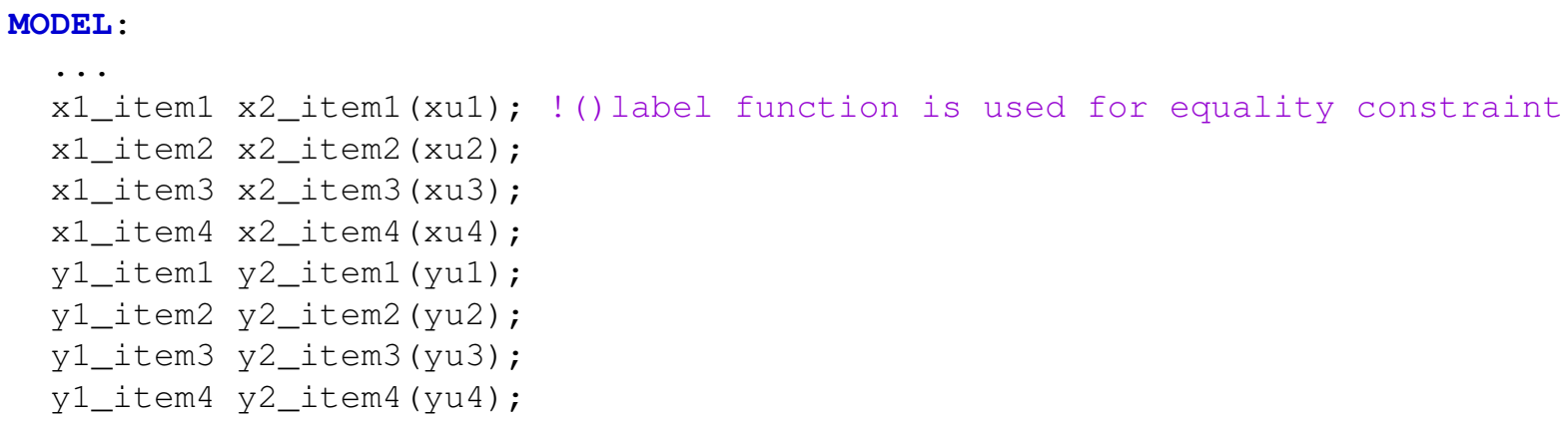

et al., 2015). The final MPLUS syntax for the measurement model can be found on the journal's website.

\section{Merging two methods: CFA-ME/I combined to the APIM with SEM}

The same stepwise procedure for testing the APIM, presented in Fitzpatrick et al. (2015) article, can now be used within the confines of a structural equation model that includes the equality constraints from the test of measurement invariance. The APIM is used for testing dyadic patterns that might emerge when analyzing dyadic data. This model tests both actor and partner effects, in which each delineates the effect one has on his or her own outcome (i.e., actor effect) and the influence one has on their partner's outcome (i.e., partner effect). Dyadic patterns can be tested within the APIM with the $k$ parameter that was first introduced by (Kenny \& Ledermann, 2010). The $k$ parameter is a ratio of the partner effect on the actor effect $(k=p / a)$. Accordingly, the general APIM has two $k$ parameters that can be estimated, one for each dyad member. As much as the APIM procedure allows for the testing of different hypotheses, it is important to understand how this procedure may be merged with existing statistical analyses - measurement invariance - in order to efficiently and reliably utilize all the data at hand.

As such, the three steps from our past article (Fitzpatrick et al., 2015) will be used to estimate the APIM with latent variables: (1) Looking for non-trivial actor effects; (2) estimating the $k$ parameters with bootstrapping; (3) interpreting $k$ and the underlying dyadic pattern. Moreover, following the footprints of Kenny (2013), this article will extend the interpretation of the $k$ parameters with valuable information that will help researchers better interpret their data.

As explained by Kenny (2013), the $k$ parameter value is estimated and then fixed to an interpretable value, depending on the generated confidence intervals. Those values are 1, 0, -1 or even 0.5. Following this logic, we wish to include more interpretable values that will augment the chance of finding an interpretable dyadic pattern in the 
data and render the interpretation even more precise. If a $k$ parameter of 0.5 denotes a partner effect that is half the size of the actor effect, we can therefore include more values like $1.5,2,-1.5$ and -2 in order to compare and interpret results. A $k$ of 1.5 would represent a partner effect that is one and a half times larger than the actor effect; a $k$ of 2.0 would represent a partner effect that is twice as large as the actor effect. Overall, these additional values should be understood as the representation of the dyadic pattern occurring in the APIM. The estimated actor and partner effects offer much of the information needed for interpretation. However, the $k$ parameters add important value to this interpretation as it helps test specific dyadic pattern hypotheses. Interestingly, more nuanced interpretations can be found when we seek to understand any given $k$ parameter. $^{1}$

\section{APIM structural steps}

(1) Looking for non-trivial actor effects. The first step to the APIM procedure is to test a saturated model with zero degrees of freedom and verify that actor effects are significant. However, models with latent variables can be globally identified but locally saturated. In our example of the APIM with latent variables, the model is globally identified with 106 degrees of freedom. However, the structural part of the model is saturated because all the paths are estimated between the latent variables. Therefore, this model is comparable to the basic saturated APIM estimated with manifest variables. Listing 6 indicates how to fit this model.

As seen in the bottom part of Table 1, the basic APIM with latent variables provided a good fit to the data. All actor and partner effects are significant in our example (a1 = $0.31, \mathrm{a} 2=0.52, \mathrm{p} 12=0.42, \mathrm{p} 21=0.27)$. Interestingly, if we compare those effects with an APIM that does not model latent variables with invariance testing, effects are smaller than the latent variable model. Table 2 shows the parameter values of this and the Step $4 \mathrm{~b}$ models. They are now stronger in magnitude because modeling latent variables corrects for measurement errors. Furthermore, these new estimates are more reliable because they were obtained in a model that assumes invariance of the latent variables. Based on these estimations we can proceed to the next step: all actor and partner effects were significant, which allows us to compute ratio parameters that will be interpretable with dyadic patterns.

(2) Estimating the $k$ parameters with bootstrapping. We then estimate the $k$ parameters in the structural model with the use of phantom variables as presented in Fitzpatrick et al.'s (2015) article. Please note that bootstrapped
95\% CIs cannot be estimated with the maximum likelihood robust estimator, so we used the normal maximum likelihood estimation but only to estimate the bootstrapped CIs. However, the overall estimation of the model was performed using maximum likelihood robust in order to obtain fit indices and parameter estimates that take into account the non-normality of the data. It is worth noting that the estimation of the $k$ parameter is not affected by the chosen estimator. Listing 7 provides the instructions.

This model had good fit indices and is an equivalent model to the basic APIM model (Table 2). In our example, $k_{1}$ was 1.496 with bias-corrected (BC) 95\% CI [0.4288.476] and $k_{2}$ was 0.459 , BC 95\% CI [0.069-1.154]. This final model can be seen in Figure 1 with detailed information on the invariance specification done in prior steps.

(3) Interpreting $k$ and the underlying dyadic patterns. Lastly, we have to interpret our findings. We have therefore updated the flowchart that was presented in Fitzpatrick et al.'s (2015) article with new interpretable values for the $k$ parameter. This updated flowchart is shown in Figure 2. In our example, both $k$ parameters included more than one interpretable value within their bootstrapped CIs ( $k_{1}$ of $0.5,1.0$ and $1.5 ; k_{2}$ of 1.0 and 0.5 ). In similar situations - which are likely to be quite frequent - we propose to settle with the nearest interpretable value of the parameter estimate of $k$, rather than solely relying on the upper and lower boundaries of the CIs. These interpretations are substantially important because they offer a more precise and nuanced interpretation of the dyadic patterns. According to the bootstrapped CIs, while considering the nearest interpretable value to the estimate, we fixed $k_{1}$ to 1.5 and $k_{2}$ to 0.5 , as seen in Listing 8 .

Based on the $\Delta S-B \chi^{2}$, this new model was not significantly different than the previous, as seen in Table 1; last line of the table, Step 4b. Males generally displayed a couple-oriented pattern, given the significance of the actor and the received partner effect. However, the $k_{1}$ ratio of 1.5 suggests that the couple-oriented pattern of the male is characterized by a preponderant partner effect 1.5 times stronger than the actor effect. The effect of the women's sensitivity on their own relationship satisfaction is two times stronger than her received partner effect (i.e., $X 1 \leftarrow Y 2$ ). Each member is positively influenced by their own level of sensitivity and their partner's sensitivity, but the preponderance of the actor to partner effect depicts within-dyad variations.

The final interpretation of this new integrative APIM model with latent variables is similar to the APIM with manifest variables (Table 2). The most important aspect to consider is that with the use of ME/I, we minimize the

\footnotetext{
${ }^{1}$ The $k$ values are not limited between -2 and 2, however it is better to look for values that are interpretable and meaningful which are in line with dyadic patterns.
} 
Table 2 - Difference between the parameters of an APIM with manifest variables and latent variables

\begin{tabular}{lccc}
\hline & Manifest variables APIM & Latent variable with invariance APIM & Differences between the two methods \\
\hline$c_{1}$ & .088 & .106 & 0.018 \\
$c_{2}$ & $.267^{*}$ & $.271^{*}$ & 0.004 \\
$a_{1}$ & $.244^{*}$ & $.311^{*}$ & 0.067 \\
$a_{2}$ & $.403^{*}$ & $.518^{*}$ & 0.115 \\
$p_{21}$ & $.387^{*}$ & $.416^{*}$ & 0.029 \\
$p_{12}$ & $.200^{*}$ & $.266^{*}$ & 0.066 \\
$k_{1}$ & 1.739 & 1.496 & 0.018 \\
& {$[0.544-10.868]$} & {$[0.428-8.476]$} & 0.004 \\
$k_{2}$ & 0.454 & 0.459 & \\
& {$[0.028-1.124]$} & {$[0.069-1.154]$} & \\
\hline
\end{tabular}

Note. Note. In [] are the $95 \%$ confidence interval. $* p<.05$.

Listing 6 - Model command for the saturated APIM. These commands follow those of Listings 2, 4 and 5.

MODEL :

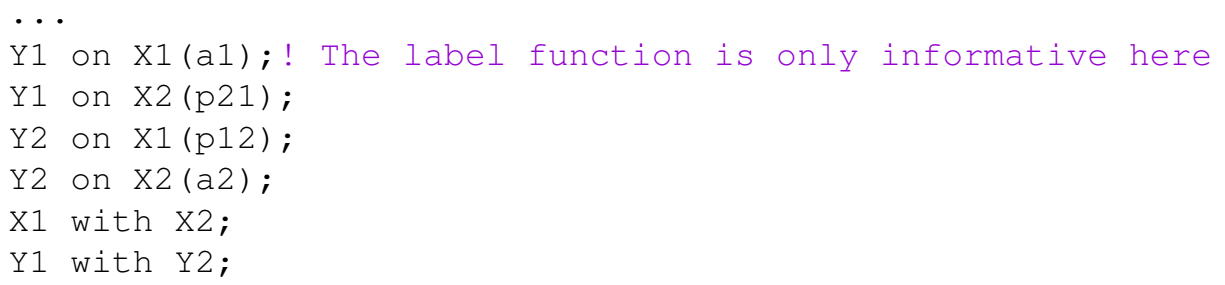

risk that the differential effects of males and females could be attributable to "comparing apples to sandwiches to sand wedges”. An APIM with manifest variables is likely to underestimate the strength of the actor and partner effects, which could, in some circumstances, result in concluding that effects are trivial. Therefore, APIM with latent variables provide estimates of the true actor effects and the true partner effects. Consequently, the k parameters in the APIM with latent variables are likely to be more precise and trustworthy. The addition of the CFA ME/I in the framework of the APIM offers a critical extension to explore the sources of non-invariance in order to fully disclose and inform other researchers about the measurement contexts in which the actor and partner effects were estimated.

\section{Discussion}

The main goal of this article was to present an integrative method for testing the APIM using structural equation modeling. We proposed a new way of approaching APIM testing by acknowledging possible divergences in the factor structure with the ME/I hypothesis testing. Dyadic data brings important considerations to light about the equality of constructs. We proposed adding a procedure of invariance to any APIM as a means to diminish the uncertainty on the equality of constructs. Moreover, following the foot- prints of Kenny (2013), we extend the interpretation of the $k$ parameter with valuable information that will help researchers better interpret their data. As demonstrated, the ratio parameters (i.e., $k$ parameters) of the actor and partner effects hold a broader interpretation of values that are meaningful. Invariance testing is an important procedure when the same measures are administered more than once. As in a longitudinal dataset, when different individuals are nested in dyads it is important to test in statistical term the equality of the measures so that the observed effect is not attenuated.

In recent years, there have been considerable advances in addressing issues of invariance in dyadic data. Chiorri, Day, and Malmberg (2014) utilized Bayesian structural equation modeling approximate measurement invariance (BSEM-AMI) analysis in order to test mean differences in romantic partner's self-report of relationship-level variables. This article shows how BSEM-AMI is able to identify the lack of support for a strong invariance model (i.e., a model that assumes that all intercepts are perfectly invariant across partners) and release the assumption of zero differences between intercepts (Chiorri et al., 2014). As a result, models showed an acceptable fit and this pointed to the existence of differences between partner's scores on a same measure. Van Dulmen and Goncy (2010) have also 
Listing 7 analysis, Model and Ouput command for estimating the $k$ parameters in APIM. These commands complement Listing ?? for the ANALYSIS command and replace 6 for the MODEL command, and complement Listing 3 for the OUTPUT command.

\section{ANALYSIS :}

...

MODEL :

BOOTSTRAP $=5000 ;$

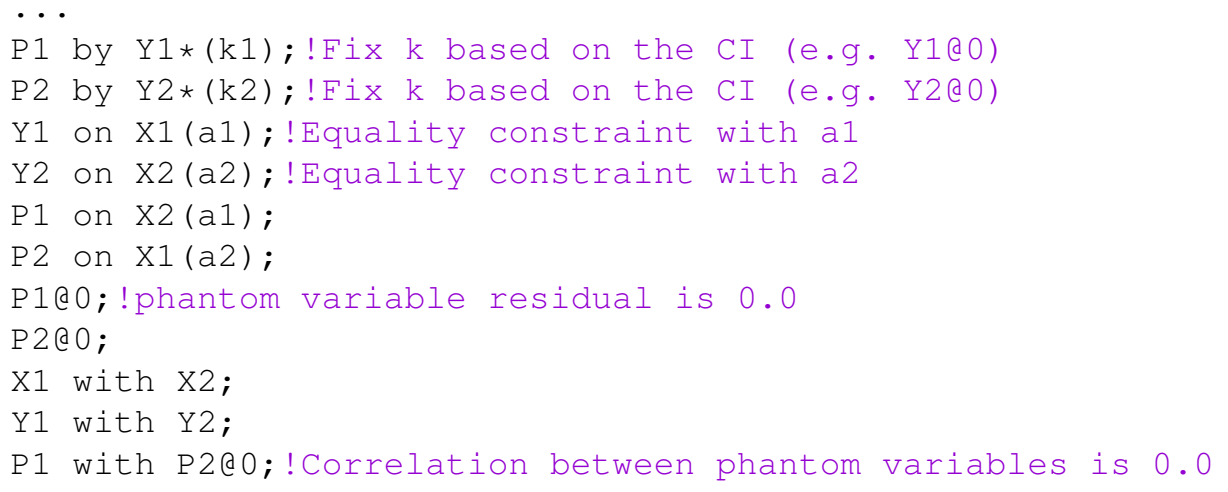

recently made advances in the field by providing an extension of the APIM within a path analysis framework by incorporating cross-informant data on the outcome variable. This technique takes into consideration reports from both romantic partners on their own and each other's behavioural functioning, which may increase the precision of the measured construct. A similar study conducted by Orth (2013) pointed to the importance of cross-informant data in dyadic data analyses. Specifically, Orth (2013) utilized a model that controlled for shared method variance. As it is mentioned in Orth's (2013) paper, the importance of this method is even greater when modeling an APIM since self-report measures may inflate actor effects.

\section{Conclusion}

There have been great efforts in the past years for incorporating statistical methods that aim at modeling dyadic data. The current article searched to bridge the gap between standard parsimonious APIM modelling with current statistical techniques that offer more precise interpretation of the data. Modeling the APIM with latent variables enables researchers to carefully examine the measurement invariance of their constructs across members of the dyad before estimating actor and partner effects by taking into account measurement error. Moreover, the invariance testing described in this article could also be applied to other dyadic models, such as the common fate model, the mutual influence model, and even the mediational or moderation forms of the APIM. Future methodological research should try to expand the APIM in order to facilitate the investigation of socially important and theoretically-driven research questions.

\section{Authors' note}

This study was supported by a scholarship from the Social Sciences and Humanities Research (SSHRC) council awarded to the first and second author. 
Figure 1 - Results for the structural model step 2 of APIM. Non-standardized estimates are in italics, standard errors are in parentheses and standardized estimates are in bold. ${ }^{*} \mathrm{p}<.05$. Invariance specifications. (1.a) Loadings of both items 1 are fixed to 1.0. (1.b) Intercepts of both items 1 are fixed to 0.0. (1.c) Factor means are freely estimated. (1.d) Like item's uniqueness are freely estimated and correlated with each other. (2) Like item's loadings are constrained to be equal. (3) Like item's intercepts are constrained to be equal. (4) Like item's uniqueness' are constrained to be equal.

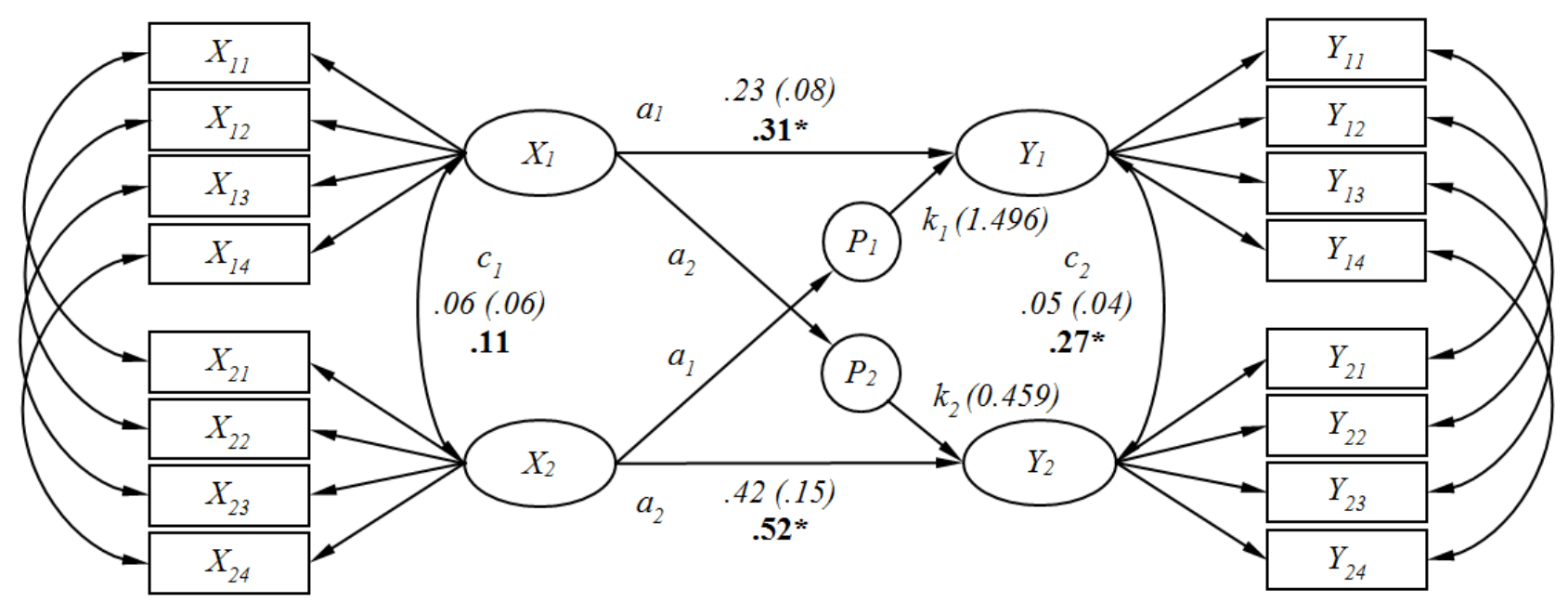

\section{References}

Bolger, N. \& Shrout, P. E. (2007). Accounting for statistical dependency in longitudinal data on dyads. In T. D. Little, J. A. Bovaird, \& N. A. Card (Eds.), Modeling ecological and contextual effects in longitudinal studies of human development (pp. 285-298). Mahwah, NJ, US: Lawrence Erlbaum Associates Publishers.

Brown, T. A. (2006). Confirmatory factor analysis for applied research. New York, NY, US: Guilford Press.

Cheung, G. W. \& Rensvold, R. B. (1999). Testing factorial invariance across groups: a reconceptualization and proposed new method. Journal of Management, 25, 127. doi:10.1177/014920639902500101

Chiorri, C., Day, T., \& Malmberg, L.-E. (2014). An approximate measurement invariance approach to withincouple relationship quality. Frontiers in psychology, 5. doi:10.3389/fpsyg.2014.00983

Colwell, S. R. (2013). Chi-square difference testing using the satorra-bentler scaled chi-square. Retrieved, 26. Retrieved from http://www.uoguelph.ca/

Cook, W. L. (1994). A structural equation model of dyadic relationships within the family system. Journal of Consulting and Clinical Psychology, 62, 500-509. doi:10.1037/0022-006X.62.3.500

Fitzpatrick, J., Gareau, A., Gaudreau, P., \& Lafontaine, M.-F. (2015). How to use the actor-partner interdependence model (apim) to estimate different dyadic patterns in mplus: a step-by-step tutorial. The Quantitative Methods for Psychology, 12(1), 74-86.

Gunnell, K., Gareau, A., \& Gaudreau, P. (2016). Introduction to factor analysis and structural equation modeling. In N. Ntoumanis \& N. Myers (Eds.), An introduction to intermediate and advanced statistical analyses for sport and exercise scientists (pp. 79-100). Wiley.

Kenny, D. A. (2013). Using $\mathrm{k}$ for estimate and test patterns in apim. Retrieved, 17. Retrieved from http:// davidakenny.net/webinars/Dyad/k/k.html

Kenny, D. A. \& Ledermann, T. (2010). Detecting, measuring, and testing dyadic patterns in the actor-partner interdependance model. Journal of Family Psychology, 24, 359-366. doi:10.1037/a0019651

Kunce, L. J. \& Shaver, P. R. (1994). An attachmenttheoretical approach to caregiving in romantic relationships. In K. J. Bartholomew \& D. Perlman (Eds.), Attachment processes in adulthood (pp. 205-237). London: Jessica Kingsley Publishers.

Meade, A. W., Johnson, E. C., \& Braddy, P. W. (2008). Power and sensitivity of alternative fit indices in tests of measurement invariance. Journal of applied psychology, 93, 568-592. doi:10.1037/0021-9010.93.3.568

Millsap, R. E. \& Yun-Tein, J. (2004). Assessing factorial invariance in ordered-categorical measures. Multivari-

The Quantitative Methods for Psychology 
Figure 2 - Flow chart for interpreting the $k$ parameters

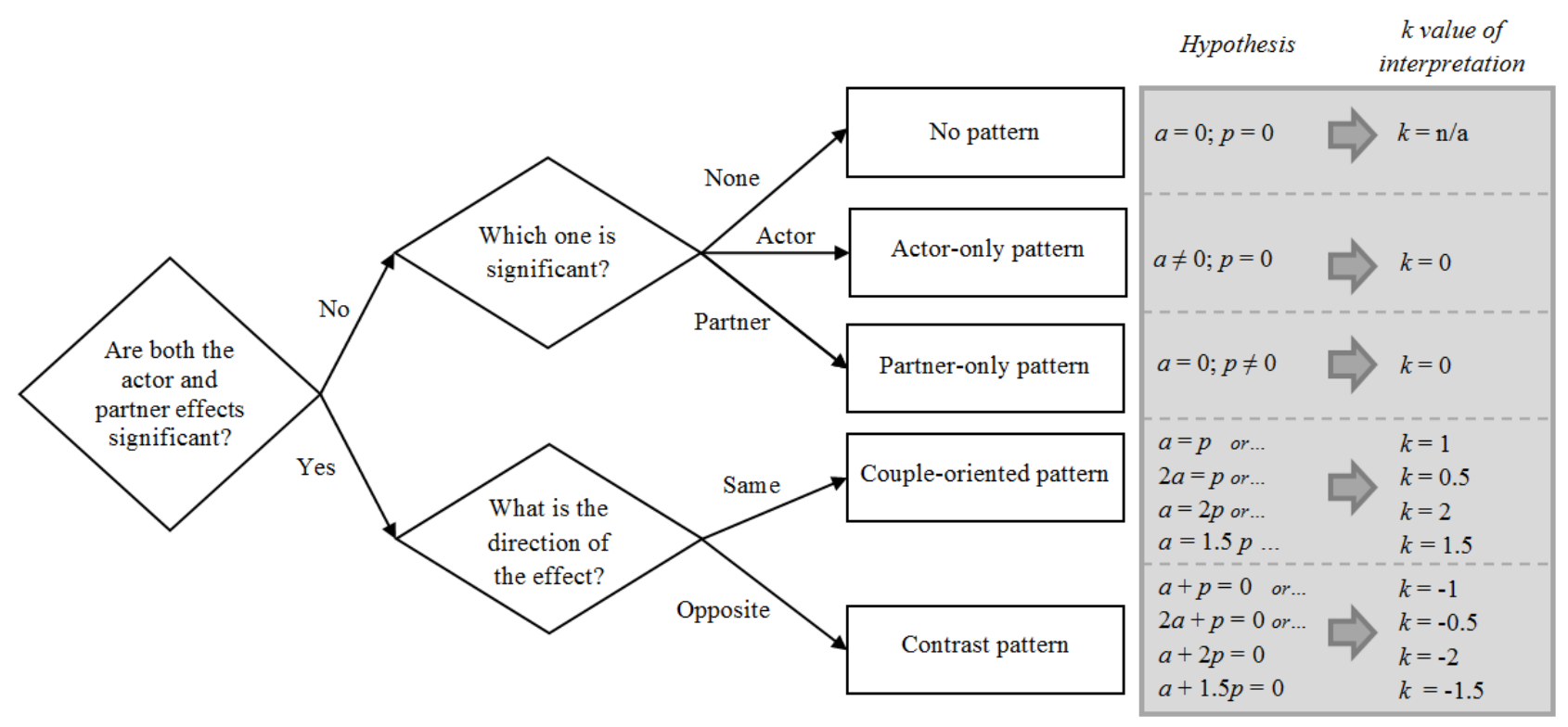

ate Behavioral Research, 39, 479-515. doi:10 . 1207 / S15327906MBR3903_4

Orth, U. (2013). How large are actor and partner effects of personality on relationship satisfaction? the importance of controlling for shared method variance. Personality \& social psychology bulletin, 39, 1359-1372. doi:10.1177/0146167213492429

Sabourin, S., Valois, P., \& Lussier, Y. (2005). Development and validation of a brief version of the dyadic adjustment scale with a nonparametric item analysis model. Psychological Assessment, 17, 15-27. doi:10 . 1037/1040-3590.17.1.15

Satorra, A. \& Bentler, P. M. (1994). Corrections to test statistics and standard errors in covariance structure analysis. In von Eye \& C. C. Clogg (Eds.), A (pp. 399-419). Latent variables analysis: Applications for developmental research . Thousand Oaks, CA, US: Sage Publications, Inc.

Satorra, A. \& Bentler, P. M. (2001). A scaled difference chisquare test statistic for moment structure analysis. Psychometrika, 66, 507-514. doi:10.1007/Bf02296192
Soper, D. (2013, January). Statistics calculators. Retrieved, 15, 2014. Retrieved from http://www danielsoper. com/statcalc3/calc.aspx?id=11

Van Dulmen, M. H. \& Goncy, E. A. (2010). Extending the actor-partner interdependence model to include cross-informant data.J Adolesc, 33(6), 869-877. doi:10. 1016/j.adolescence.2010.07.002

Vandenberg, R. J. \& Lance, C. E. (2000). A review and synthesis of the measurement invariance literature: suggestions, practices, and recommendations for organizational research. Organizational Research Methods, 3, 4-70. doi:10.1177/109442810031002

Weston, R. \& Gore, P. A. (2006). A brief guide to structural equation modeling. Counseling Psychologist, 34, 719751. doi:10.1177/0011000006286345

Yong, A. G. \& Pearce, S. (2013). A beginner's guide to factor analysis: focusing on exploratory factor analysis. Tutorials in Quantitative Methods for Psychology, 9(2), 79-94.

\section{Open practices}

(1) The Open Data badge was earned because the data of the experiment(s) are available on the journal's web site.

- The Open Material badge was earned because supplementary material(s) are available on the journal's web site. 
Listing 8 - Model command for estimating the final APIM. The MODEL command in Listing 7 should be replaced by these commands.

MODEL :

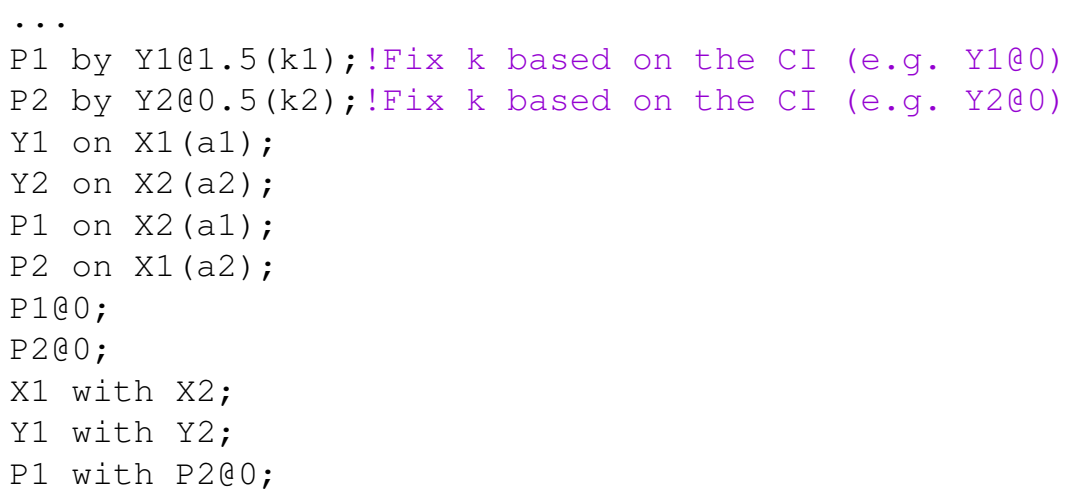

\section{Citation}

Gareau, A., Fitzpatrick, J., Gaudreau, P., \& Lafontaine, M.-F. (2016). Analysing, interpreting, and testing the invariance of the actor-partner interdependence model. The Quantitative Methods for Psychology, 12(2), 101-113. doi:10.20982/ tqmp.12.2.p101

Copyright (C) 2016, Gareau, Fitzpatrick, Gaudreau, Lafontaine . This is an open-access article distributed under the terms of the Creative Commons Attribution License (CC BY). The use, distribution or reproduction in other forums is permitted, provided the original author(s) or licensor are credited and that the original publication in this journal is cited, in accordance with accepted academic practice. No use, distribution or reproduction is permitted which does not comply with these terms.

Received: 01/01/2016 Accepted: 04/03/2015 